\title{
On detecting discrete Cheshire charge *
}

\author{
Martin Bucher, Kai-Ming Lee and John Preskill \\ California Institute of Technology, Pasadena, CA 91125, USA \\ Received 23 December 1991 \\ (Revised 25 May 1992) \\ Accepted for publication 26 May 1992
}

\begin{abstract}
We analyze the charges carried by loops of string in models with non-abelian local discrete symmetry. The charge on a loop has no localized source, but can be detected by means of the Aharonov-Bohm interaction of the loop with another string. We describe the process of charge detection, and the transfer of charge between point particles and string loops, in terms of gauge-invariant correlation functions.
\end{abstract}

\section{Introduction}

In a spontaneously broken gauge theory, if the unbroken gauge group $\mathrm{H}$ is a discrete subgroup of the underlying continuous gauge group $\mathrm{G}$, then the theory will contain topologically stable strings (in $3+1$ dimensions) or vortices (in $2+1$ dimensions). If $\mathrm{H}$ is non-abelian, the strings have remarkable properties. In particular, a closed loop of string can carry a non-trivial H-charge. Oddly, this charge is a global property of the string that can not be attributed to any locally defined charge density. Yet the charge is physically detectable, for the charged string loop has an infinite-range Aharonov-Bohm interaction with other strings. Furthermore, if a pointlike particle carrying $\mathrm{H}$-charge winds through a string loop, the particle and the loop can exchange charge.

Charge with no localized source has been called "Cheshire charge" [1]. It was first discussed for the case of the "Alice" string [2]. A loop of Alice string can carry electric charge, and have a long-range electric field, even though the electric charge density vanishes everywhere $[1,3,4]$. Processes in which electric (or magnetic) charge is exchanged between string loops and point particles were discussed in refs. [1,4-6].

In this paper, we analyze the purely quantum mechanical version of Cheshire charge $[4,7]$ that arises in a theory with a non-abelian discrete local H-symmetry $[8,9]$. The semiclassical theory of discrete Cheshire charge was formulated in ref. [4], and elaborated in refs. $[10,11]$. Here we extend the theory further, in several

* This work supported in part by the US Department of Energy under Contract DE-AC03-81-ER40050 
respects. We describe how a charge operator can be constructed, such that the expectation value of the operator in a state specifies the transformation properties of the state under global H-transformations. We then study processes in which charge is exchanged between string loops and point particles, and derive general formulas for how the expectation value of the charge of the loop is altered by the exchange. Finally, we explain how the charge exchange processes can be probed using gauge-invariant correlation functions.

The charge operator and correlation functions are also treated in ref. [12], where lattice realizations of operators and correlators are extensively discussed.

The rest of this paper is organized as follows: In sect. 2, we briefly review the basic properties of non-abelian strings and the concept of Cheshire charge. We construct the non-abelian charge operator in sect. 3 , and analyze the charge exchange process in sect. 4 . Sect. 5 contains some final comments.

\section{Non-abelian strings}

Let us briefly recall some of the properties of non-abelian strings in three spatial dimensions (and vortices in two spatial dimensions).

If a simply connected gauge group $G$ is broken to a discrete subgroup $H$, then strings are classified by elements of $\mathrm{H}$. To assign a group element to a loop of string, we fix an (arbitrary) basepoint $x_{0}$, and specify a path $C$, beginning and ending at $x_{0}$, that winds once through the string loop. (See fig. 1.) The assigned group element is then

$$
a\left(C, x_{0}\right)=P \exp \left(i \int_{C, x_{0}} A \cdot \mathrm{d} x\right)
$$

We refer to $a\left(C, x_{0}\right)$ as the "flux" of the string; it encodes the effect of parallel transport around the path $C$. The flux takes values in $\mathrm{H}\left(x_{0}\right)$, the subgroup of $\mathrm{G}$ that stabilizes the Higgs condensate at the point $x_{0}$ (since parallel transport around $C$ must return the condensate to its original value). Since $H$ is discrete, the

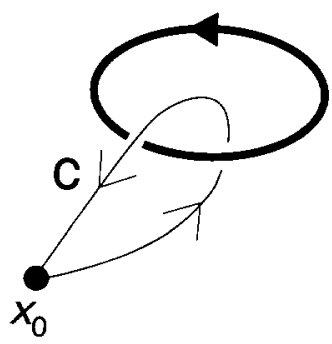

Fig. 1. The path $C$, starting and ending at the point $x_{0}$, encircles a loop of string. 
flux $a\left(C, x_{0}\right)$ is unchanged by deformations of $C$ that leave $x_{0}$ fixed, as long as $C$ never crosses the core of the string.

For a configuration of many string loops, we specify a standard path for each loop, where all paths have the same basepoint. Evidently, the flux associated with the product path $C_{2} \circ C_{1}$ obtained by traversing first $C_{1}$ and then $C_{2}$ is just the product $a\left(C_{2}, x_{0}\right) \cdot a\left(C_{1}, x_{0}\right)$ of the two fluxes associated with $C_{1}$ and $C_{2}$. Thus, $a\left(C, x_{0}\right)$ defines a homomorphism that maps $\pi_{1}\left(\mathscr{M}, x_{0}\right)$ to $\mathrm{H}$, where $\mathscr{M}$ is the manifold that is obtained when the cores of all strings are removed from $\mathbb{R}^{3}$.

The flux assigned to a path is not gauge invariant. The gauge transformations at the basepoint $x_{0}$ that preserve the condensate at the basepoint, and so preserve the embedding of $\mathrm{H}$ in $\mathrm{G}$, take values in $\mathrm{H}\left(x_{0}\right)$. Under such a gauge transformation $h \in \mathrm{H}\left(x_{0}\right)$, the flux transforms as

$$
a\left(C, x_{0}\right) \rightarrow h a\left(C, x_{0}\right) h^{-1}
$$

In a many-string configuration, the flux of each string becomes conjugated by $h$.

In the presence of strings, the embedding of the unbroken group $H$ in $G$ necessarily depends on the spatial position $x$. If the strings are non-abelian, this position dependence is described by a non-trivial fiber bundle. The base space of the bundle is the spatial manifold $\mathscr{M}$, the fiber is $\mathrm{H}$, and the structure group is also $\mathrm{H}$, which acts on the fiber by conjugation. The bundle is twisted: Upon transport around the path $C$, the group element $h \in \mathrm{H}\left(x_{0}\right)$ becomes conjugated by $a\left(C, x_{0}\right)$. This twist prevents the bundle from being smoothly deformed to the trivial bundle $\mathscr{M} \times \mathrm{H}$. One thus says that the unbroken H-symmetry is not "globally realizable" $[1,4,13]$; there is no smooth function of position that describes how the unbroken group is embedded in $\mathrm{G}$. Only the subgroup of $\mathrm{H}$ that commutes with the flux of all strings is globally realizable on $\mathscr{M}$.

To define the H-charge of a state, we will want to consider how the state transforms under global H-transformations. Fortunately, these global gauge transformations can be implemented, even though a topological obstruction prevents $H$ from being globally realized. The point is that it is sufficient to be able to define an $\mathrm{H}$-transformation on and outside a large surface $\Sigma$ (homeomorphic to $\mathrm{S}^{2}$ ) that encloses all of the string loops. The transformation cannot be smoothly extended inside the sphere if it is required to take values in $H(x)$. However, one may relax this requirement and allow the gauge transformation to take values in $G$ inside of $\Sigma$; then a smooth extension is possible. It makes no difference what extension is chosen, for gauge transformations of compact support act trivially on physical states. (In two spatial dimensions, the only global H-transformations that can be implemented are those that commute with the total flux; i.e. the flux associated with a path that encloses all of the vortices.)

If the basepoint $x_{0}$ lies outside the surface $\Sigma$, then, under the global gauge transformation $h \in \mathrm{H}$, the flux of a string transforms as in eq. (2.2). Thus, the 
H-representations mix up the string loop state labeled by $a \in \mathrm{H}$ with string loop states labeled by other group elements in the same conjugacy class as $a$. Let $[a]$ denote the conjugacy class that contains $a$. The action of $\mathrm{H}$ on the members of the class $[a]$ defines a (reducible) representation that we denote as $D^{([a])}$. In $D^{([a])}$, each element of $\mathrm{H}$ is represented by a permutation of the class, according to

$$
D^{([a])}(h):\left|a^{\prime}\right\rangle \rightarrow\left|h a^{\prime} h^{-1}\right\rangle, \quad a^{\prime} \in[a] .
$$

This representation can be decomposed into irreducible representations of $\mathrm{H}$. For each class $[a]$ there is a unique state that can be constructed that transforms trivially under $\mathrm{H}$; it is the superposition of flux eigenstates

$$
|0 ;[a]\rangle=\frac{1}{\sqrt{n_{[a]}}} \sum_{a^{\prime} \in[a]}\left|a^{\prime}\right\rangle,
$$

where $n_{[a]}$ denotes the order of the class. The other states contained in the decomposition of $D^{([a])}$ carry H-charge. This is "discrete Cheshire charge," for the charge of the loop has no localized source. (Note that the charged string states transform trivially under the center of $\mathrm{H}$, since $D^{([a])}$ represents the center trivially).

The splitting between the charge- 0 string state (2.4) and the lowest charge excitation of the string is of order $\exp (-\kappa A)$, where $\kappa$ is a string tension, and $A$ is the area of the string loop $[11,12]$. It is a remarkable property of Cheshire charge that, in the presence of a large string loop, the gap between the ground state and the first charged excitation is much less than the corresponding gap when the string is absent. Indeed, the gap approaches zero very rapidly as the size of the loop increases.

The above discussion of the discrete Cheshire charge carried by a string loop also applies, in two spatial dimensions, to the charge carried by a vortex pair, if the total flux of the pair is trivial. In fact, in two dimensions, there is a somewhat more general notion of Cheshire charge. We may consider a pair of vortices with flux $a_{1}$ and $a_{2}$, such that the total flux $a_{\text {tot }}=a_{1} a_{2}$ is a non-trivial element of $H$. Then the global gauge transformations that can be implemented belong to the normalizer $\mathrm{N}\left(a_{\text {tot }}\right)$, the subgroup of $\mathrm{H}$ that commutes with the total flux. Unless $a_{1}$ and $a_{2}$ commute with all elements of $\mathrm{N}\left(a_{\text {tot }}\right)$, the two-vortex states will transfrom as a non-trivial representation of $\mathrm{N}\left(a_{\text {tot }}\right)$, which can be decomposed into its irreducible components. Thus, there are two-vortex states that carry non-trivial $\mathrm{N}\left(a_{\text {tot }}\right)$-charge.

\section{Charge operator}

The discrete charge of an object, including a charged string loop, can be detected at long range by means of the Aharonov-Bohm effect [14]. Let $|u\rangle$ 
denote the wave function in internal-symmetry space of an object located at $x_{0}$ that transforms as the irreducible representation $D^{(\nu)}$ of $\mathrm{H}$. Then when the particle is transported around the closed path $C$ that begins and ends at $x_{0}$, the wave function is modified according to

$$
|u\rangle \rightarrow D^{(\nu)}\left[a\left(C, x_{0}\right)\right]|u\rangle .
$$

If the string is in the flux eigenstate $|a\rangle$, then the Aharonov-Bohm phase that can be measured in an interference experiment is

$$
\left\langle u\left|D^{(\nu)}(a)\right| u\right\rangle .
$$

But if the string is in the charge-zero eigenstate $|0 ;[a]\rangle$ given by eq. (2.4), then the expectation value of the "phase" $D^{(\nu)}(a)$ becomes

$$
\frac{1}{n_{[a]}} \sum_{a^{\prime} \in[a]} D^{(\nu)}\left(a^{\prime}\right)=\frac{1}{n_{\mathrm{H}}} \sum_{h \in \mathrm{H}} D^{(\nu)}\left(h a h^{-1}\right)=\frac{1}{n_{\nu}} \chi^{(\nu)}(a) \mathbb{1},
$$

where $n_{\mathrm{H}}$ is the order of the group, $n_{\nu}$ is the dimension of $D^{(\nu)}$, and $\chi^{(\nu)}$ is the character of the representation. The second equality follows from Schur's lemma.

In principle, the charge inside a large region can be measured by means of a process in which the world-sheet of a string sweeps over the boundary of the region. If the string is in the charge-zero eigenstate $|0 ;[a]\rangle$, and the object enclosed by the world-sheet transforms as the irreducible representation $(\nu)$ of $\mathrm{H}$, then the amplitude for this process will be weighted by the Aharonov-Bohm factor $\left(1 / n_{\nu}\right) \chi^{(\nu)}(a)$. The charge $(\nu)$ of an unidentified object can be determined by measuring this factor for each class $[a]$.

A gauge-invariant operator $F_{[a]}(\Sigma)$ can be constructed that inserts, as a classical source, a string world-sheet in the state $|0 ;[a]\rangle$ on the closed surface $\Sigma$. The realization of this operator in a euclidean lattice gauge theory was described in ref. [4] in the case where $\mathrm{H}$ is abelian (see also ref. [15]), and in refs. [12,16] for $\mathrm{H}$ non-abelian. (It is closely related to the 't Hooft loop operator [17].) If the surface $\Sigma$ is chosen to lie in a time-slice, then the operator $F_{[a]}(\Sigma)$ measures the charge enclosed by $\Sigma$. To define the charge of an isolated object, we consider a surface $\Sigma$ that encloses the object, and whose closest approach to the object is large compared to the correlation length of the theory. Let $|\psi\rangle$ denote the quantum state of the object. Then we have

$$
\frac{\left\langle\psi\left|F_{[a]}(\Sigma)\right| \psi\right\rangle}{\left\langle F_{[a]}(\Sigma)\right\rangle_{0}}=\sum_{\nu} p^{(\nu)}(\psi ; \Sigma) \frac{1}{n_{\nu}} \chi^{(\nu)}(a),
$$

where $p^{(\nu)}(\psi ; \Sigma)$ is the probability that the object carries charge $(\nu)$. By measuring $F_{[a]}(\Sigma)$ for each class, we can determine all of the $p^{(\nu)}$. (It is necessary to divide by 
the vacuum expectation value $\left\langle F_{[a]}(\Sigma)\right\rangle_{0}$ to remove the effects of quantum mechanical vacuum charge fluctuations near the surface $\Sigma$ [4].)

The Aharonov-Bohm interaction makes it possible to detect $\mathrm{H}$-charge at arbitrarily long range. Thus, a theory with discrete local $\mathrm{H}$-symmetry obeys a charge superselection rule - no gauge-invariant local operator can create or destroy H-charge. We have

$$
\langle(\mu)|\mathscr{O}|(\nu)\rangle=0, \quad(\mu) \neq(\nu),
$$

where $\mathscr{\sigma}$ is any local observable, and $|(\nu)\rangle$ denotes a state that transforms as the irreducible representation $(\nu)$ of $\mathrm{H}$. We can construct a projection operator that projects out a given superselection sector of the Hilbert space. It is

$$
P^{(\nu)}=\frac{n_{\nu}}{n_{\mathrm{H}}} \sum_{a \in \mathrm{H}} \chi^{(\nu)}(a)^{*} U(a),
$$

where $U(a)$ represents the global $\mathrm{H}$-transformation $a \in \mathrm{H}$ acting on the Hilbert space. This projection operator can be expressed in terms of the operators $F_{[a]}(\Sigma)$, for it follows from eq. (3.4) that

$$
\frac{F_{[a]}(\Sigma)}{\left\langle F_{[a]}(\Sigma)\right\rangle_{0}} \rightarrow \frac{1}{n_{[a]}} \sum_{a^{\prime} \in[a]} U\left(a^{\prime}\right),
$$

as the surface $\Sigma$ approaches the surface at spatial infinity.

We can also use the operator $F_{[a]}$ to construct an "Aharonov-Bohm Order Parameter" (ABOP) that probes whether non-trivial superselection sectors actually exist. Let

$$
W^{(\nu)}(C) \equiv \frac{1}{n_{\nu}} \chi^{(\nu)}\left[P \exp \left(i \int_{C} A \cdot \mathrm{d} x\right)\right]
$$

denote the Wilson loop operator in the irreducible representation $(\nu)$. This operator introduces a classical source with charge $(\nu)$ propagating on the world-line $C$. The ABOP is defined by

$$
A_{[a]}^{(\nu)}(\Sigma, C) \equiv \frac{F_{[a]}(\Sigma) W^{(\nu)}(C)}{\left\langle F_{[a]}(\Sigma)\right\rangle_{0}\left\langle W^{(\nu)}(C)\right\rangle_{0}} .
$$

If $\mathrm{H}$ quantum numbers can indeed be detected at infinite range, then we expect that

$$
\left\langle A_{[a]}^{(\nu)}(\Sigma, C)\right\rangle_{0} \rightarrow \frac{1}{n_{\nu}} \chi^{(\nu)}\left(a^{k(\Sigma, C)}\right)
$$


in the limit in which $\Sigma$ and $C$ increase to infinite size, with the closest approach of $\Sigma$ to $C$ also approaching infinity. Here $k(\Sigma, C)$ is the linking number of the surface $\Sigma$ and the loop $C$. (In the abelian case, the ABOP was first described in ref. [15], and was further elaborated in ref. [4]. The non-abelian generalization was introduced in ref. [16], and its properties were extensively discussed in ref. [12].)

In two spatial dimensions, the classification of superselection sectors is richer than in three dimensions $[4,12]$. The Aharonov-Bohm interaction makes it possible to measure the magnetic flux of a vortex (up to conjugation) at arbitrarily long range; therefore, no local observable can change the conjugacy class of the total magnetic flux. The magnetic flux (up to conjugation) of an object can be measured by the Wilson loop operator $W^{(\nu)}(C)$. We choose the loop $C$ to lie in a time-slice, such that its closest approach to the object is large compared to the correlation length of the theory. Then

$$
\frac{\left\langle\psi\left|W^{(\nu)}(C)\right| \psi\right\rangle}{\left\langle W^{(\nu)}(C)\right\rangle_{0}}=\sum_{[a]} p_{[a]}(\psi ; C) \frac{1}{n_{\nu}} \chi^{(\nu)}(a),
$$

where $p_{[a]}(\psi ; C)$ is the probability that the state $|\psi\rangle$ has flux $[a]$. By measuring $W^{(\nu)}(C)$ for each irreducible representation $(\nu)$, we can determine all of the $p_{[a]}$.

As we noted in sect. 2 , if the total flux of a state is $a_{\text {tot }}$, then the charge of the state is specified by an irreducible representation of the normalizer $N\left(a_{\text {tot }}\right)$. This is because the only global gauge transformations that can be implemented acting on this state are elements of the normalizer. In more physical terms, the charge of an object is measured by means of its Aharonov-Bohm interaction with a distant test-vortex. Such a measurement can be carried out only if the flux of the test vortex commutes with the flux of the object. Otherwise, the flux of the test vortex changes when it circumnavigates the object, due to the holonomy interaction $[10,18]$, and this obscures the interference pattern. Thus, superselection sectors are labeled by the class $\left[a_{\text {tot }}\right]$ of the total flux, and an irreducible representation of the normalizer $\mathrm{N}\left(a_{\text {tot }}\right)$. (The abstract group $\mathrm{N}\left(a_{\text {tot }}\right)$ is independent of how the class representative $a_{\text {tot }}$ is chosen.)

The operators $F_{[a]}(\Sigma)$ and $A_{[a]}^{(\nu)}(\Sigma, C)$ can also be constructed in two spatial dimensions. Then $\Sigma$ becomes a closed curve that can be interpreted as the world-line of a vortex-antivortex pair. But for the purpose of measuring the charge of a state in two dimensions, it is convenient to generalize the construction of the operator $F_{[a]}(\Sigma)$. If the state has non-trivial flux, the charge is a representation of the normalizer of the flux, and there is a charge-zero vortex pair associated with each class of the normalizer. There are gauge-invariant operators that, in effect, insert such charge-zero pairs (as classical sources) on the world-line $\Sigma$.

To construct these operators, we must specify a basepoint $x_{0}$. Then a projection operator $P_{(a)}\left(\Sigma, x_{0}\right)$ can be constructed that projects out states that have flux $a$ associated with the path $\Sigma$ that begins and ends at $x_{0}$. There is also an operator 
$\tilde{F}_{(b)}\left(\Sigma, x_{0}\right)$ that introduces a flux eigenstate vortex pair on $\Sigma$, where the flux $b$ is defined with respect to paths that begin and end at $x_{0}$, These operators are not invariant under $\mathrm{H}$ gauge transformations; under a gauge transformation $h \in \mathrm{H}\left(x_{0}\right)$, they transform as

$$
h: \quad P_{(a)} \rightarrow P_{\left(h a h^{-1}\right)}, \quad \tilde{F}_{(b)} \rightarrow \tilde{F}_{\left(h b h^{-1}\right)} .
$$

But the combination

$$
F_{[a, b]}\left(\Sigma, x_{0}\right) \equiv \frac{1}{n_{\mathbf{H}}} \sum_{h \in \mathbf{H}} P_{\left(h a h^{-1}\right)}\left(\Sigma, x_{0}\right) \tilde{F}_{\left(h b h^{-1}\right)}\left(\Sigma, x_{0}\right)
$$

is gauge invariant. (It is unchanged if $a$ and $b$ are conjugated by the same element of $\mathrm{H}$, and is also independent of how the basepoint $x_{0}$ is chosen.) Were it not for the flux projection operators, the left-hand side of eq. (3.13) would be just the operator $F_{[b]}$. And, indeed, if $a$ is the identity $e$, we have

$$
F_{[e, b]}=F_{[b]} P_{(e)}
$$

just our old $F$ operator times a projection onto the trivial flux sector.

Now suppose that $\Sigma$ lies in a time-slice, and that we evaluate the expectation value of $F_{[a, b]}(\Sigma)$ in a state with non-trivial flux. Suppose, to be specific, that the flux contained inside $\Sigma$ is in the same $H$ conjugacy class as $a$. Then the projection operator will restrict the sum over $h$ to the elements of the normalizer of the flux. Furthermore, the contribution to the expectation value due to terms in which $h b h^{-1}$ does not commute with the flux will be heavily suppressed when $\Sigma$ is large and far from the object that is being measured. To see this, note that, if a vortex-antivortex pair with flux $b^{\prime}$ winds around a vortex with flux $a^{\prime}$, and $a^{\prime}$ and $b^{\prime}$ do not commute, then the pair cannot re-annihilate unless flux is exchanged between the pair and the $a^{\prime}$ vortex. (This consequence of the holonomy interaction was discussed in ref. [12].) In effect, then, $h b h^{-1}$ is restricted to the normalizer of the flux of the state (because the other contributions are suppressed by $\tilde{F}$ ), and ranges over precisely one class of the normalizer (because of the projection operator $P$ ). Therefore, if the state $\left|[a],\left(\nu_{a}\right)\right\rangle$ transforms as the irreducible representation $\left(\nu_{a}\right)$ of the normalizer $\mathrm{N}(a)$, and if $a$ and $b$ commute, we find

$$
\frac{\left\langle[a],\left(\nu_{a}\right)\left|F_{[a, b]}(\Sigma)\right|[a],\left(\nu_{a}\right)\right\rangle}{\left\langle F_{[a, b]}(\Sigma)\right\rangle_{0}}=\frac{1}{n_{\left(\nu_{a}\right)}} \chi^{\left(\nu_{a}\right)}(b), \quad b \in \mathrm{N}(a) .
$$

But if $a$ and $b$ do not commute, or if the flux of the state is not in the class $[a]$, then

$$
\frac{\left\langle[c],\left(\nu_{c}\right)\left|F_{[a, b]}(\Sigma)\right|[c],\left(\nu_{c}\right)\right\rangle}{\left\langle F_{[a, b]}(\Sigma)\right\rangle_{0}}=0, \quad c \notin[a] \text { or } b \notin \mathrm{N}(a)
$$


(in the limit where $\Sigma$ becomes very large). By measuring the expectation value of $F_{[a, b]}$ for all commuting $a$ and $b$, we can determine the flux and charge of a state.

\section{Charge transfer}

We will now consider the non-abelian Aharonov-Bohm interactions between string loops and point particles, and demonstrate that exchange of $\mathrm{H}$-charge can occur.

The total H-charge of a composite system consisting of a string loop and a charged particle can be measured by studying the Aharonov-Bohm interaction of the composite with other, much larger, string loops. Obviously, then, the total $\mathrm{H}$-charge of the composite must be conserved; it cannot change when the particle winds through the loop. Charge exchange is an inevitable consequence of charge conservation.

To see this, it is convenient to imagine a composite of a string loop and a particle-antiparticle pair, where, initially, both the loop and the pair have zero charge (transform trivially under $\mathrm{H}$ ). Suppose that the particle transforms as the irreducible representation $D^{(\nu)}$ of $\mathrm{H}$; the antiparticle transforms as the conjugate representation. Let $\left\{e_{i}^{(\nu)} \mid i=1,2, \ldots, n_{\nu}\right\}$ denote an orthonormal basis for the vector space on which $D^{(\nu)}$ acts. Then the initial state of the pair has the group-theoretic structure

$$
\left|\psi_{\mathrm{in}}^{(\nu)}\right\rangle=\frac{1}{\sqrt{n_{\nu}}}\left|e_{i}^{(\nu) *} \otimes e_{i}^{(\nu)}\right\rangle
$$

(summed over $i$ ). The initial state of the loop is the state $|0 ;[a]\rangle$ defined in eq. (2.4).

Suppose that the particle and antiparticle are initially at the point $x_{0}$. Then the particle traverses a path $C$ that winds through the string loop and returns to $x_{0}$. After this traversal, the state of the pair and the state of the loop are correlated. The total charge is still zero, but in general the pair and the loop both have a non-trivial charge. We can infer the final charge on the loop by calculating the final charge carried by the pair. In fact, the final charge of the pair is actually independent of the initial charge of the loop; it depends only on the class $[a]$. Thus, to calculate the final charge of the pair, we may take the state of the loop to be the flux eigenstate $|a\rangle$ (where the flux is defined in terms of the path $C$ as in eq. (2.1)). It does not matter how the class representative $a$ is chosen.

Using eq. (3.1), we find that the state of the pair after the traversal is

$$
\left|\psi_{\text {fin }}^{(\nu)}, a\right\rangle=\frac{1}{\sqrt{n_{\nu}}}\left|e_{i}^{(\nu) *} \otimes e_{j}^{(\nu)}\right\rangle D_{j i}^{(\nu)}(a) .
$$


This state $\left|\psi_{\mathrm{fin}}^{(\nu)}, a\right\rangle$ does not transform as a definite irreducible representation of $\mathrm{H}$, but it can, of course, be decomposed into states of definite $\mathrm{H}$-charge. The probability $p^{(\mu)}$ that the $\mathrm{H}$-charge is $(\mu)$ can be extracted by using the projection operator $P^{(\mu)}$ defined by eq. (3.6). We find

$$
\begin{aligned}
p_{\text {pair }}^{(\mu)}(\nu,[a])= & \left\langle\psi_{\text {fin }}^{(\nu)}, a\left|P^{(\mu)}\right| \psi_{\text {fin }}^{(\nu)}, a\right\rangle \\
= & \frac{n_{H}}{n_{\nu} n_{\mathrm{H}}} \sum_{b \in \mathrm{H}} \chi^{(\mu)}\left(b^{-1}\right) D_{n m}^{(\nu) *}(a)\left\langle e_{m}^{(\nu) *} \otimes e_{n}^{(\nu)} \mid e_{k}^{(\nu) *} \otimes e_{l}^{(\nu)}\right\rangle \\
& \times D_{k i}^{(\nu) *}(b) D_{l j}^{(\nu)}(b) D_{j i}^{(\nu)}(a) \\
= & \frac{n_{\mu}}{n_{\nu} n_{\mathrm{H}}} \sum_{b \in \mathbf{H}} \chi^{(\mu)}\left(b^{-1}\right) \chi^{(\nu)}\left(b a b^{-1} a^{-1}\right) .
\end{aligned}
$$

As we anticipated, this result is unchanged if $a$ is replaced by $a^{\prime} \in[a]$.

If the total $\mathrm{H}$-charge is zero, then the composite of string loop and pair has a wave function of the form

$$
\left.\left.\left|\psi_{[a]}^{(\nu)}\right\rangle=\sum_{\mu} \sqrt{p_{\text {pair }}^{(\mu)}} \mid \text { loop, } \mu *\right\rangle \otimes \mid \text { pair }, \mu\right\rangle
$$

Thus, the probability that the loop carries charge $(\mu)$ is given by

$$
p_{\text {loop }}^{(\mu)}(\nu,[a])=p_{\text {pair }}^{(\mu *)}(\nu,[a])=p_{\text {loop }}^{(\mu *)}(\nu *,[a])=p_{\text {loop }}^{(\mu *)}\left(\nu,\left[a^{-1}\right]\right) .
$$

Of course, this probability is non-vanishing only if $D^{(\mu)}$ is contained in $D^{(\nu) *} \otimes D^{(\nu)}$ and represents the center of $\mathrm{H}$ trivially.

We can directly verify that detectable Cheshire charge now resides on the string loop by studying an appropriate gauge-invariant correlation function. Consider the process depicted in fig. 2. This process is shown in $2+1$ dimensions for ease of visualization, but the generalization to $3+1$ dimensions is straightforward. At time $t_{1}$, a vortex-antivortex pair is created. The flux of the vortex lies in the class $[a]$, and the (initial) $\mathrm{H}$-charge of the vortex pair is trivial. At time $t_{2}$, a particle-antiparticle pair is created. The particle has H-charge $(\nu)$, and the pair is (initially) uncharged. Then the particle winds counterclockwise around the $[a]$ vortex, transferring charge to the vortex pair. Next, another vortex-antivortex pair, with flux lying in the class $[b]$, winds around the (now charged) [a] vortex pair, acquiring an Aharonov-Bohm phase that is sensitive to the charge of the $[a]$ pair. Then the charge- $(\nu)$ particle winds clockwise around the $[a]$ vortex, discharging the $[a]$ pair. Finally, the particle-antiparticle pair is annihilated at time $t_{3}$, and the $[a]$ vortex-antivortex pair is annihilated at time $t_{4}$. 


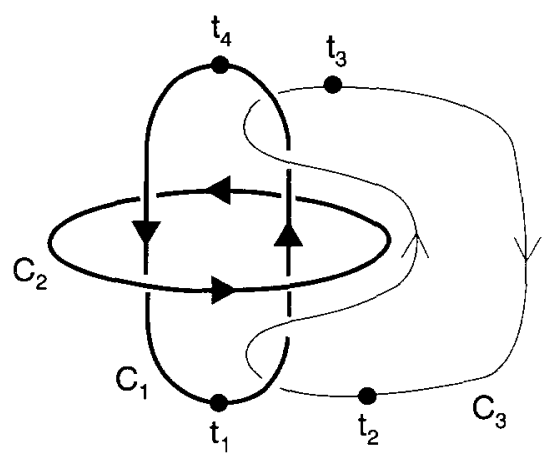

Fig. 2. The Borromean rings. $C_{1}$ is the world-line of an $[a]$ vortex, $C_{2}$ is the world-line of a $[b]$ vortex, and $C_{3}$ is the world-line of a charged particle that transforms as the representation $(\nu)$. The charged particle transfers charge to the $[a]$ vortex-antivortex pair, and the charge is subsequently detected via the Aharonov-Bohm interaction of the pair with the $[b]$ vortex.

If the vortices and charged particles are treated as classical sources, this process is described by the correlation function

$$
\left\langle F_{[a]}\left(C_{1}\right) F_{[b]}\left(C_{2}\right) W^{(\nu)}\left(C_{3}\right)\right\rangle_{0}
$$

where $C_{1}$ is the world-line of the $[a]$ vortex, $C_{2}$ is the world line of the $[b]$ vortex, and $C_{3}$ is the world-line of the charged particle. As shown in fig. 2, the three loops $C_{1}, C_{2}$ and $C_{3}$ are joined in a topologically non-trivial configuration known as the "Borromean rings" [19]; no two loops are linked, yet the loops cannot be separated without crossing.

By considering the case where the loops are large and far apart, and comparing with the case where the loops are unjoined, we can isolate the Aharonov-Bohm factor acquired by the $[b]$ vortex pair that winds around the charged $[a]$ vortex pair. The calculation of eq. (4.6), using weak-coupling perturbation theory on the lattice, is described in ref. [12]. We will not repeat the details of the calculation here, but it is easy to explain the main idea. Loosely speaking, inserting a classical vortex with flux $a$ on the closed path $C_{1}$ is equivalent to performing a singular gauge transformation on a surface $S_{1}$ that is bounded by $C_{1}$. The path has an orientation, which induces an orientation of the surface. The effect of the singular gauge transformation on the Wilson loop $W^{(\nu)}\left(C_{3}\right)$ is to insert the factor $D^{(\nu)}(a)$ where $C_{3}$ crosses $S_{1}$ in a positive sense, and to insert the factor $D^{(\nu)}\left(a^{-1}\right)$ where $C_{3}$ crosses $S_{1}$ in a negative sense. In fig. 3 , we see that the loop $C_{3}$ successively crosses $S_{2}$ in a negative sense, $S_{1}$ in a negative sense, $S_{2}$ in a positive sense, and $S_{1}$ in a positive sense, before closing. Due to the path ordering of the Wilson loop, the factor due to a later crossing appears to the left of the factor due to an earlier crossing, These crossings therefore modify $\left\langle W^{(\nu)}\left(C_{3}\right)\right\rangle_{0}$ by the factor $\left(1 / n_{\nu}\right) \chi^{(\nu)}\left(a b a^{-1} b^{-1}\right)$ compared to the case where $C_{3}$ is unjoined with $C_{1}$ and $C_{2}$. 


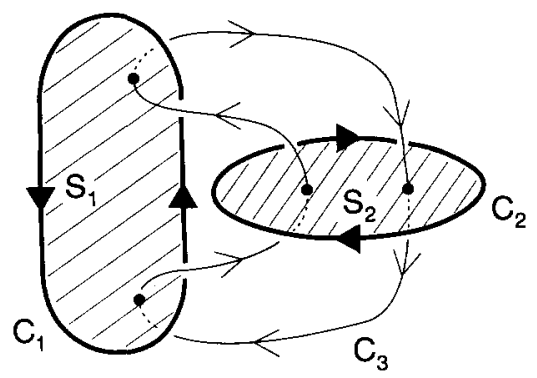

Fig. 3. A deformation of the rings shown in fig. 2. The gauge field is singular on the surfaces $S_{1}$ and $S_{2}$ that are bounded by the loops $C_{1}$ and $C_{2}$.

Recalling that $a$ and $b$ are averaged over a class when $F_{[a]}$ and $F_{[b]}$ are inserted, we find that

$$
\frac{\left\langle F_{[a]}\left(C_{1}\right) F_{[b]}\left(C_{2}\right) W^{(\nu)}\left(C_{3}\right)\right\rangle_{0}}{\left\langle F_{[a]}\left(C_{1}\right)\right\rangle_{0}\left\langle F_{[b]}\left(C_{2}\right)\right\rangle_{0}\left\langle W^{(\nu)}\left(C_{3}\right)\right\rangle_{0}} \rightarrow \frac{1}{n_{\mathrm{H}}} \sum_{h \in \mathrm{H}} \frac{1}{n_{\nu}} \chi^{(\nu)}\left(h a h^{-1} b h a^{-1} h^{-1} b^{-1}\right)
$$

when the loops are large, far apart, and joined.

In $3+1$ dimensions, there is an analog of the Borromean ring configuration, in which two disjoint closed surfaces $\Sigma_{1}$ and $\Sigma_{2}$ are joined by a closed loop $C_{3}$ that does not link with either surface. For this configuration, eq. (4.7) still applies, with $C_{1}$ and $C_{2}$ replaced by $\Sigma_{1}$ and $\Sigma_{2}$. We can decompose the right-hand side of eq. (4.7) into characters as

$$
\sum_{\mu} p_{\text {loop }}^{(\mu)}(\nu,[a]) \frac{1}{n_{\mu}} \chi^{(\mu)}(b)
$$

where $p_{\text {loop }}^{(\mu)}(\nu,[a])$ is the probability that the charge carried by the $[a]$ string loop, and detected by the $[b]$ string loop, is $(\mu)$. (Compare eq. 3.4).) Using the orthogonality of the characters, we find from eqs. (4.7) and (4.8) that

$$
p_{\text {loop }}^{(\mu)}(\nu,[a])=\frac{n_{\mu}}{n_{\nu} n_{\mathrm{H}}} \sum_{b \in \mathrm{H}} \chi^{(\mu)}\left(b^{-1}\right) \chi^{(\nu)}\left(a b a^{-1} b^{-1}\right),
$$

in agreement with eqs. (4.5) and (4.3). Thus, the charge lost by the particle pair has indeed been transferred to the $[a]$ string loop. (Note that, in order to get the right answer, it is important to choose consistent orientations for the world sheets $\Sigma_{1}$ and $\Sigma_{2}-$ the $[a]$ string must pass through the $[b]$ string in the same sense that the Wilson loop passes through the $[a]$ string. Otherwise, we would in effect be measuring the charge of the $[a]$ string with a $\left[b^{-1}\right]$ string, rather than a $[b]$ string.) 
We will now derive eq. (4.7) by a different method that invokes the "holonomy interaction" between string loops. Consider two flux-eigenstate string loops that initially carry flux $a$ and $b$. Now suppose that the $b$-loop sweeps around the $a$-loop and returns to its original position. After this process, the flux of the $b$-loop is unchanged, but the flux of the $a$-loop has been altered; it has become a $b a b^{-1}$ loop $[6,10,18]$. (Here again, we must be careful about the orientations of the string loops. The $a$-loop becomes a $b a b^{-1}$ loop if it passes through the $b$-loop in the same sense as the path $C$ that is used to define the flux of the $b$-loop. If it passes through the $b$-loop in the opposite sense, it becomes a $b^{-1} a b$ loop.)

Return now to the Borromean-ring process. Suppose that two string loops are initially in the flux eigenstate $|a, b\rangle$. Then a particle-antiparticle pair is created, and the particle winds through the $a$-loop; the new state of the string loops and the particle-antiparticle pair can be expressed as

$$
\frac{1}{\sqrt{n_{\nu}}}\left|a, b, e_{i}^{(\nu) *} \otimes e_{j}^{(\nu)}\right\rangle D_{j i}^{(\nu)}(a)
$$

(Compare eq. (4.2).) When the $b$-loop sweeps around the $a$-loop, the state becomes

$$
\frac{1}{\sqrt{n_{\nu}}}\left|b a b^{-1}, b, e_{i}^{(\nu) *} \otimes e_{j}^{(\nu)}\right\rangle D_{j i}^{(\nu)}(a)
$$

due to the holonomy interaction. Now the particle winds back through the $b a b^{-1}$ loop (in the opposite sense), and the state becomes

$$
\frac{1}{\sqrt{n_{\nu}}}\left|b a b^{-1}, b, e_{i}^{(\nu) *} \otimes e_{k}^{(\nu)}\right\rangle D_{k j}^{(\nu)}\left(b a^{-1} b^{-1}\right) D_{j i}^{(\nu)}(a) .
$$

Finally, the particle-antiparticle pair annihilates, and we have

$$
\frac{1}{n_{\nu}} \chi^{(\nu)}\left(a b a^{-1} b^{-1}\right)\left|b^{-1} a b, b\right\rangle .
$$

To reproduce eq. (4.7), we must take the initial string state to be $|0 ;[a], 0 ;[b]\rangle$, in which the $[a]$ and $[b]$ loops are both uncharged. Thus, we average both $a$ and $b$ over a class. We find that the effect of the particle-antiparticle pair on the string state is

$$
|0 ;[a], 0 ;[b]\rangle \rightarrow\left(\frac{1}{n_{\mathrm{H}}} \sum_{h \in \mathrm{H}} \frac{1}{n_{\nu}} \chi^{(\nu)}\left(h a h^{-1} b h a^{-1} h^{-1} b^{-1}\right)\right)|0 ;[a], 0 ;[b]\rangle .
$$


By creating the initial string state and annihilating the final string state, we obtain eq. (4.7).

\section{Some final comments}

We described in sect. 3 how a charge-zero string loop can be used in an Aharonov-Bohm interference experiment to measure the charge of an object. It seems that charge could also be measured using strings that are in flux eigenstates rather than charge eigenstates. This alternative measurement process was discussed in detail in ref. [11], and in some respects it is simpler than the process that we described. So our insistence on using charge-zero string loops requires a word of explanation.

Part of the answer is that the gauge-invariant charge operator that we constructed in sect. 3 can be interpreted in terms of a process involving a charge-zero loop. In this process, a tiny string loop is created, and is stretched to a large size. The loop then winds around a region, shrinks, and re-annihilates. The AharonovBohm factor acquired by the loop is sensitive to the total charge inside the region. This string loop must be in a charge-zero state, rather than a flux eigenstate, because otherwise charge conservation would forbid the creation of the loop and its subsequent annihilation.

Another point is that the notion of a flux eigenstate string suffers from an ambiguity. Of course, flux eigenstates are not energy eigenstates, so there is quantum-mechanical mixing between flux eigenstates that are in the same $\mathrm{H}$ conjugacy class. The authors of ref. [11] note that the time scale for this mixing is of order $\exp (\kappa A)$, where $\kappa$ is the string tension and $A$ is the area of the loop. Therefore, the mixing can be easily turned off by choosing the string loop to be sufficiently large.

But this is not quite the whole story, for there is another type of "mixing" that should be considered [12]. Recall that to define the flux of a string, we must choose an arbitrary "basepoint" $x_{0}$, and a path $C$, beginning and ending at $x_{0}$, that winds around the string. Suppose that a virtual string loop nucleates, lassoes the basepoint, and then re-annihilates. If the string is initially assigned the flux $a$, and the virtual string has flux $b$, then this process changes the flux to $b a b^{-1}$, due to the holonomy interaction. In physical terms, we may prepare a string in a flux eigenstate, and then measure its flux later by doing an Aharonov-Bohm interference experiment. We may find that the flux has been altered, not because of the mixing of flux eigenstates, but rather because of quantum fluctuations that are independent of the size of the loop. Of course, virtual string loops are strongly suppressed at weak coupling; the amplitude for such a process is of order $\exp \left(-C / e^{2}\right)$, where $e$ is the gauge coupling. But it is a significant point of principle that quantum fluctuations render ambiguous the notion of the flux of the 
string. No such ambiguity afflicts the conjugacy class of the flux, or the charge carried by a loop.

For these reasons, we have used charge-zero strings in our discussion of the charge measurement process. We can imagine doing a double-slit experiment with a beam of particles of unknown charge, where a string loop in the state $|0 ;[a]\rangle$ surrounds one of the slits. By observing how the shift in the interference pattern depends on the class [a], we can determine the character of the representation according to which the particles in the beam transform, and so infer their charge.

However, the phenomenon of charge transfer raises a puzzle. If a particle passes through the slit that is surrounded by the string, it transfers charge to the string. By measuring the charge on the string loop later, we can find out which slit the particle passed through. Thus, no interference pattern should be seen.

The resolution of this puzzle is that there is a non-vanishing probability, in general, that no charge transfer takes place. This probability is given by eq. (4.9) in the case where $(\mu)$ is the trivial representation $(0)$; we then have

$$
p_{\text {loop }}^{(0)}(\nu,[a])=\left|\frac{1}{n_{\nu}} \chi^{(\nu)}(a)\right|^{2}
$$

Therefore, as long as the character does not vanish, it is possible for the particle to slip through the string loop without being detected, and an interference pattern is observed. From the interference pattern, the phase of the character, as well as its modulus, can be deduced.

We thank Mark Alford, Hoi-Kwong Lo, John March-Russell, and David Wales for useful discussions.

\section{References}

[1] M. Alford, K. Benson, S. Coleman, J. March-Russell and F. Wilczek, Phys. Rev. Lett. 64 (1990) 1632; [Erratum: 65 (1990) 668]

[2] A.S. Schwarz, Nucl. Phys. B208 (1982) 141;

A.S. Schwarz and Y.S. Tyupkin, Nucl. Phys. B209 (1982) 427

[3] P. Ginsparg and S. Coleman, unpublished (1982)

[4] J. Preskill and L. Krauss, Nucl. Phys. B341 (1990) 50

[5] M. Alford, K. Benson, S. Coleman, J. March-Russell and F. Wilczek, Nucl. Phys. B349 (1991) 414

[6] M. Bucher, H.-K. Lo and J. Preskill, Nucl. Phys. B386 (1992) 3

[7] M. Alford, J. March-Russell and F. Wilczek, Nucl. Phys. B337 (1990) 695

[8] F. Bais, Nucl. Phys. B170 (1980) 32

[9] T.W.B. Kibble, Phys. Rep. 67 (1980) 183

[10] M. Bucher, Nucl. Phys. B350 (1991) 163

[11] M. Alford, S. Coleman and J. March-Russell, Nucl. Phys. B351 (1991) 735

[12] M. Alford, K.-M. Lee, J. March-Russell and J. Preskill, Quantum field theory of non-abelian strings and vortices, Caltech preprint CALT-68-1700 (1991), Nucl. Phys. B384 (1992) 251

[13] A. Balachandran, F. Lizzi and V. Rogers, Phys. Rev. Lett. 52 (1984) 1818 
[14] L. Krauss and F. Wilczek, Phys. Rev. Lett. 62 (1989) 1221

[15] K. Fredenhagen and M. Marcu, Commun. Math. Phys. 92 (1983) 81;

K. Fredenhagen, Particle structure of gauge theories, in Fundamental problems of gauge field theory, ed. G. Velo and A.S. Wightman (Plenum, New York, 1986)

[16] M. Alford and J. March-Russell, Nucl. Phys. B369 (1992) 276

[17] G. 't Hooft, Nucl. Phys. B138 (1978) 1; B153 (1979) 141

[18] F. Wilczek and Y.-S. Wu, Phys. Rev. Lett. 65 (1990) 13

[19] D. Rolfsen, Knots and links (Publish or Perish, Wilmington, 1976) 